Zofia SADOWSKA

Netting materials

\title{
RELATIONSHIP BETWEEN KNOT SLIP RESISTANCE AND NATURE OF YARN SURFACE
}

\section{ZALEŻNOŚĆ ODPORNOŚCI NA PRZESUWANIE WĘZEÓW TKANIN SIECIOWYCH OD CHARAKTERU POWIERZCHNI PRZĘDZ}

\author{
Institute of Aquaculture and Fisheries Technology, Academy of \\ Agriculture, Szczecin
}

\begin{abstract}
An effect of the yarn surface nature upon knot slip resistance, the latter serving as a measure of fishing nets' knot stability, was studied. The yarn surface character was described by the friction coefficient of the yarn, determined at a specially designed measuring unit. The results were treated statistically. The nature of ydrn surface was found to affect considerably the knot slip resistance.
\end{abstract}

\section{INTRODUCTION}

The fishing gear currently in use are manufactured of various textile materials (yarns, twines and cordage, webbing). The nature of surface of textile products depends primarily on fibre properties and surface texture. Force of friction, termed also friction, is the physical quantity used to characterise yarn surfaces in manufacturing and application of textile products, netting materials included (Grudniewski, 1973).

The literature dealing with studies on netting materials' surface characters is scant. At the same time the question of the yarn surface effect upon the knot stability in a fishing net has become very relevant to the manufacturing of synthetic knotted nettings. The studies carried. out by Dembiński (1956), Lomakina (1959), and Kalinowski (1962) concerned the influence of roughness, hardness, and friction coefficient of yarns on the knot stability in fishing nets. The results indicate these factors to bear a clear effect upon the stability. 
Within 1971-1973, netting yarns supplied by the home and overseas monofilament producers were in use in Poland. When undertaking the studies described below it was hoped, basing on investigations carried out on these materials (Sadowska, 1976, 197.7), that synthetic (polyamide and polyester) yarns supplied by various prodưcers and differing in their chemicals properties would exhibit different properties of their surfaces. The present work was aimed at following the relationship between the knot stability of fishing nets and the character of synthetic yarn surfaces, the knot stability being measured as the knot slip resistance.

\section{MATERIALS AND METHODS}

\section{Materials}

Synthetic (polyamide and polyester) netting yarns and nets machine-made of these yarns were the materials to be studied. Cabled netting yarns were produced of single ones made in Poland (stylon NL, torlen) and abroad (amilan - Japan, enkalon - Holland). The following sorts of polyamide yarns were subject to the studies: 23 tex x $1 \times 2,23$ tex $\mathrm{x} 4 \times 3,23$ tex $\times 8 \times 3$; the polyester ones studied comprised the 28 tex $1 \times 2$ and 110 tex x 1 x 3 yarns. Eleven samples of "raw" (no physico-chemical finishing treatment) nettings were woven for the purpose of the present study. Each grade of yarns served to produce simultaneously, on a net weaving loom, webbings of stylon, enkalon, amilan, and torlen. The nominal mesh size was $40 \mathrm{~mm}$.

\section{Methods}

Due to the lack of a specialised equipment, yarn friction coefficients were determined on a measuring unit designed and built at the Fishing Techniques Department. The unit was based on a ZT-40 tensile testing machine into which an additional dynamometer with crocodile clamps and ball bearing-mounted block were fitted, thus any bias of the results caused by friction on the block being eliminated.

Yarn friction coefficients were measured from two yarns, one immobile and the other shifting upon it. This procedure enables a kinetic friction coefficient to be calculated from measurements of tension $\left(\mathrm{P}_{0}\right)$ in one yarn, tension plus friction force $\left(\mathrm{P}_{1}\right)$ in the other, and the angle under which the yarns ends can be clamped $(\varphi)$ (Grudniewski, 1973; Godek, 1973). The friction coefficient is calculated from Euler's formula.

The measurements were performed both on pre-adjusted and wet yarns. A total number of 20 measurements were made. A loop-shaped section of a yarn (B) was fastened to the upper clamp of the tensile testing machine. One end of the other yarn (A) was clamped in the dynamometer, the section of the yarn being led over the block and through the loop B, while the other end of the yarn was fastened to the testing machine lower clamp. The yarn A, after leaving the loop was prevented from touching the yarn section preceeding the loop. The measuring system thus prepared served to read. the values of $P_{0}$ and $P_{1}$. The $P_{0}$ values were calculated for each sort of yarn in relation to the 
resultant actual number expressed as tex. $P_{0}$ expressed as $\mathrm{kG}$ equalled $5 \mathrm{G} /$ tex for a given sort. The tensile testing machine turned on, a shift of the yarn A along the yarn B occurred.

The lower clamp move velocity was $100 \mathrm{~mm} / \mathrm{min}$. When the dynamometer showed the assumed $P_{o}$ value $(k G)=5 G /$ tex, the value of $P_{1}$ was read on the machine dynamometer. Euler's formula describes the relationship between $\mathrm{P}_{0}$ and $\mathrm{P}_{1}$ as

$$
\mathrm{P}_{1}=\mathrm{P}_{\mathrm{o}} \cdot \mathrm{e}^{\mu \varphi}
$$

where:

$$
\begin{aligned}
& \mathrm{P}_{1} \text { and } \mathrm{P}_{0}=\text { operating forces } \\
& \mu \varphi=\text { friction coefficients } \\
& \varphi=\text { the angle }(\mathrm{rd})
\end{aligned}
$$

In the measuring system used, the forces $P_{0}$ and $P_{1}$ operated parallel to each other, the angle of the yarn A on B was assumed $180^{\circ}$. The friction coefficient is calculated from the transformed Euler's formula:

$$
\mu=\frac{1}{\varphi} \ln \frac{\mathrm{P}_{1}}{\mathrm{P}_{0}}
$$

The factory-produced netting knot slip resistance was studied basing on the method outlined in the Polish Standard PN-69/P-85036; the tests were performed on ZT-20 and ZT-40 tensile testing machines. The knot slip resistance was analysed from 50 knots of each sort randomly taken out of a sheet of a factory-made webbing. The measurements were made at the ambient atmosphere (temperature ca $20^{\circ} \mathrm{C}$, humidity ca $70-80 \%$ ) as well as following the 24 hrs distilled water soaking.

The resulting reading of the knot slip resistance consisted of a value of a force $(\mathrm{kG})$ deforming the knot so that the warp yarn slipped out of the knot or the yarn was ruptured in the knot.

\section{Processing and evaluation of the results}

The friction coefficient was calculated for every measurement according to the transformed Euler's formula (2) and given as the arithmetic mean of measurements taken. The knot slip resistance index was calculated as the arithmetic mean. Standard deviation (s) and coefficient of variation (v) were calculated for the abovementioned indices. When evaluating the results of friction coefficient measurements and knot slip resistance, the analysis of variance was used. In order to assess the significance of differences between the means, multiple-mean variance and Fisher-Snedecor's test ( $F$ test) were made use of. To detect the means differing from each other as well as to assess the significance of these differences, Duncan's new multiple range test was taken advantage of. Generally available tables of critical values for particular coefficients were referred to when analysing the parameters. 


\section{RESULTS OF STUDIES}

\section{Results of studies on knot slip resistance}

These results are summarised in Table 1. Differing resistances were found in knots made of synthetic yarns of a similar number but supplied by different producers. Torlen knots display a clearly higher resistance to slippage.

\section{Results of studies on yarn coefficient of friction}

The values of yarn-on-yarn kinetic ceofficient of friction $(\mu)$ were calculated as in the formula (2) and summarised in Tables 2 and 3 for pre-adjusted and wet yarns, respectively.

Basing on the friction coefficient mean values for the pre-adjusted yarns supplied by the same producer (Table 2) it can be said that the polyamide yarn coefficients are similar and equal 0.35 for stylon, ekalon, and 0.36 for amilan. The following section will treat the problem in detail. Also the coefficients of variation (v) calculated for the analysed yarns' friction coefficients are maintained at a similar level. The kinetic friction coefficient of torlen yarns is higher, averaging 0.40 . The variation coefficient (v) of polyester yarn friction coefficient remains at the level recorded for the polyamide yarns.

The friction coefficients of wet yarns (Table 3) are lower than those of the pre-adjusted ones. This relationship, however, is not supported by the data on the polyester yarns which, when wet, display higher coefficients.

\section{DISCUSSION OF RESULTS}

In order to follow a relationship between knot slip resistance and yarn properties, two chemical groups of materials were chosen, the yarns differing in their thickness (i.e., number) and supplied by various producers. It was hoped that such selection of materials would elucidate the effect of yarn surface character on stability of knots. The analysis of variance and $F$ test show a significant difference between knot stablities of various yarns of the same of similar number (Sadowska, 1976). The difference in stabilites between torlen and polyamide knots is highly significant as shown by Duncan's procedure (Sadowska, 1976).

The studies on the kinetic friction coefficient made use of Euler's formula $(1,2)$, rather frequently applied in technical literature, as well as of a specially designed measuring unit (Fig. 1). As it is shown by the results (Table 2), the polyester yarns have a higher friction coefficient. $F$ test and Duncan's new multiple range procedure showed the differences to be highly significant (Table 4 a. 5). No unequivocal answer was obtained for the polyamide yarns of a similar number and supplied by various producers. Mean friction coefficients of the 23 tex $\times 4 \times 3$ yarns show no difference. On the other hand, among the 23 tex $\times 8 \times 3$ group there is a highly significant difference between the amilan and stylon friction coefficients ( 0.36 and 0.34 , respectively), while a significant difference exists between the amilan and enkalon coefficients $(0.36$ and 0.35 , respecti- 
Resistance to slippage $\left(\overline{\mathrm{F}}_{\mathrm{p}}\right)$ of pre-adjusted knots in machine-made "raw" nettings

\begin{tabular}{|c|c|c|c|c|c|c|c|}
\hline \multicolumn{3}{|c|}{ Sort of netting } & $\begin{array}{l}\text { Knot slip } \\
\text { resistance }\end{array}$ & $\begin{array}{c}\text { Number of } \\
\text { measurements }\end{array}$ & $\begin{array}{l}\text { Standard } \\
\text { deviation }\end{array}$ & $\begin{array}{l}\text { Variation } \\
\text { coefficient }\end{array}$ & $\begin{array}{l}\text { Standard } \\
\text { error }\end{array}$ \\
\hline & 1 & & 2 & 3 & 4 & 5 & 6 \\
\hline stylon NL & $\begin{array}{l}23 \text { tex } \\
23 \text { tex } \\
23 \text { tex }\end{array}$ & $\begin{array}{l}x 1 \times 2 \neq 40 \\
\times 4 \times 3 \neq 40 \\
\times 8 \times 3 \neq 40\end{array}$ & $\begin{array}{l}0.67 \\
1.27 \\
2.17\end{array}$ & $\begin{array}{l}50 \\
50 \\
50\end{array}$ & $\begin{array}{l}0.2635 \\
0.6336 \\
0.7513\end{array}$ & $\begin{array}{l}38.80 \\
49.60 \\
34.56\end{array}$ & $\begin{array}{r}10.97 \\
14.03 \\
9.77\end{array}$ \\
\hline enkalon & $\begin{array}{l}23 \text { tex } \\
23 \text { tex } \\
23 \text { tex }\end{array}$ & $\begin{array}{l}x 1 \times 2 \neq 40 \\
x 4 \times 3 \neq 40 \\
x 8 \times 3 \neq 40\end{array}$ & $\begin{array}{l}0.58 \\
1.68 \\
2.04\end{array}$ & $\begin{array}{l}50 \\
50 \\
50\end{array}$ & $\begin{array}{l}0.20 \\
0.8215 \\
1.0912\end{array}$ & $\begin{array}{l}34.48 \\
48.80 \\
53.43\end{array}$ & $\begin{array}{r}9.75 \\
13.80 \\
15.11\end{array}$ \\
\hline amilan & $\begin{array}{l}23 \text { tex } \\
23 \text { tex } \\
23 \text { tex }\end{array}$ & $\begin{array}{l}x 1 \times 2 \neq 40 \\
\times 4 \times 3 \neq 40 \\
x 8 \times 3 \neq 40\end{array}$ & $\begin{array}{l}0.70 \\
1.50 \\
2.64\end{array}$ & $\begin{array}{l}50 \\
50 \\
50\end{array}$ & $\begin{array}{l}0.2211 \\
0.8480 \\
1.0548\end{array}$ & $\begin{array}{l}31.42 \\
56.66 \\
39.77\end{array}$ & $\begin{array}{r}8.88 \\
16.02 \\
11.25\end{array}$ \\
\hline torlen & $\begin{array}{r}28 \text { tex } \\
110 \text { tex }\end{array}$ & $\begin{array}{l}x 1 \times 2 \neq 40 \\
x 1 \times 3 \neq 40\end{array}$ & $\begin{array}{l}1.53 \\
3.20\end{array}$ & $\begin{array}{l}50 \\
50\end{array}$ & $\begin{array}{l}0.170 \\
2.8964\end{array}$ & $\begin{array}{l}11.11 \\
90.62\end{array}$ & $\begin{array}{r}3.14 \\
25.63\end{array}$ \\
\hline
\end{tabular}


Kinetic yarn-on-yarn friction coefficient $(\mu)$ as determined with

$P_{0}=5 \mathrm{G} /$ tex for pre-adjusted yarns

\begin{tabular}{|c|c|c|c|c|c|c|}
\hline \multicolumn{2}{|r|}{ Sort of yarn } & \multirow{2}{*}{$\begin{array}{c}\begin{array}{c}\text { Friction } \\
\text { coefficient }\end{array} \\
2\end{array}$} & \multirow{2}{*}{$\begin{array}{r}\text { Group } \\
\text { mean } \\
3\end{array}$} & \multirow{2}{*}{$\begin{array}{c}\begin{array}{c}\text { Number of } \\
\text { measurements } \\
\text { (n) }\end{array} \\
4\end{array}$} & \multirow{2}{*}{$\begin{array}{c}\text { Standard } \\
\text { deviation } \\
(\mathrm{s})\end{array}$} & \multirow{2}{*}{$\begin{array}{c}\begin{array}{c}\text { Variation } \\
\text { coefficient } \\
\text { (v) }\end{array} \\
6\end{array}$} \\
\hline & 1 & & & & & \\
\hline stylon NL & $\begin{array}{l}23 \text { tex } \times 1 \times 2 \\
23 \text { tex } \times 4 \times 3 \\
23 \text { tex } \times 8 \times 3\end{array}$ & $\begin{array}{l}0.36 \\
0.35 \\
0.34\end{array}$ & 0.35 & $\begin{array}{l}20 \\
20 \\
20\end{array}$ & $\begin{array}{l}0.008 \\
0.010 \\
0.009\end{array}$ & $\begin{array}{l}2.13 \\
2.74 \\
2.76\end{array}$ \\
\hline enkalon & $\begin{array}{l}23 \text { tex } \times 1 \times 2 \\
23 \text { tex } \times 4 \times 3 \\
23 \text { tex } \times 8 \times 3\end{array}$ & $\begin{array}{l}0.36 \\
0.35 \\
0.35\end{array}$ & 0.35 & $\begin{array}{l}20 \\
20 \\
20\end{array}$ & $\begin{array}{l}0.009 \\
0.006 \\
0.010\end{array}$ & $\begin{array}{l}2.45 \\
1.80 \\
2.82\end{array}$ \\
\hline amilan & $\begin{array}{l}23 \text { tex } \times 1 \times 2 \\
23 \text { tex } \times 4 \times 3 \\
23 \text { tex } \times 8 \times 3\end{array}$ & $\begin{array}{l}0.37 \\
0.35 \\
0.36\end{array}$ & 0.36 & $\begin{array}{l}20 \\
20 \\
20\end{array}$ & $\begin{array}{l}0.012 \\
0.008 \\
0.013\end{array}$ & $\begin{array}{l}3.27 \\
2.34 \\
3.66\end{array}$ \\
\hline torlen & $\begin{array}{r}28 \text { tex } \times 1 \times 2 \\
110 \text { tex } \times 1 \times 3\end{array}$ & $\begin{array}{l}0.41 \\
0.39\end{array}$ & 0.40 & $\begin{array}{l}20 \\
20\end{array}$ & $\begin{array}{l}0.014 \\
0.011\end{array}$ & $\begin{array}{l}3.53 \\
2.84\end{array}$ \\
\hline
\end{tabular}


Kinetic yarn-on-yarn friction coefficient as determined with $P_{0}=5 \mathrm{G} /$ tex for wet yarns

\begin{tabular}{|c|c|c|c|c|c|c|}
\hline & rt of yarn & $\begin{array}{l}\text { Friction } \\
\text { coefficient }\end{array}$ & $\begin{array}{l}\text { Group } \\
\text { mean }\end{array}$ & $\begin{array}{l}\text { Number of } \\
\text { measurements } \\
\text { (n) }\end{array}$ & $\begin{array}{l}\text { Standard } \\
\text { deviation } \\
\text { (s) }\end{array}$ & $\begin{array}{l}\text { Variation } \\
\text { coefficient } \\
\text { (v) }\end{array}$ \\
\hline & 1 & 2 & 3 & 4 & 5 & 6 \\
\hline stylon NL & $\begin{array}{l}23 \text { tex } \times 1 \times 2 \\
23 \text { tex } \times 4 \times 3 \\
23 \text { tex } \times 8 \times 3\end{array}$ & $\begin{array}{l}0.34 \\
0.32 \\
0.32\end{array}$ & 0.33 & $\begin{array}{l}20 \\
20 \\
10\end{array}$ & $\begin{array}{l}0.007 \\
0.009 \\
0.005\end{array}$ & $\begin{array}{l}2.14 \\
2.87 \\
1.65\end{array}$ \\
\hline enkalon & $\begin{array}{l}23 \text { tex } \times 1 \times 2 \\
23 \text { tex } \times 4 \times 3 \\
23 \text { tex } \times 8 \times 3\end{array}$ & $\begin{array}{l}0.37 \\
0.33 \\
0.34\end{array}$ & 0.35 & $\begin{array}{l}20 \\
20 \\
20\end{array}$ & $\begin{array}{l}0.008 \\
0.009 \\
0.007\end{array}$ & $\begin{array}{l}2.29 \\
2.81 \\
2.02\end{array}$ \\
\hline amilan & $\begin{array}{l}23 \text { tex } \times 1 \times 2 \\
23 \text { tex } \times 4 \times 3 \\
23 \text { tex } \times 8 \times 3\end{array}$ & $\begin{array}{l}0.35 \\
0.34 \\
0.33\end{array}$ & 0.34 & $\begin{array}{l}20 \\
20 \\
20\end{array}$ & $\begin{array}{l}0.010 \\
0.010 \\
0.010\end{array}$ & $\begin{array}{l}2.77 \\
2.74 \\
2.09\end{array}$ \\
\hline torlen & $\begin{array}{r}28 \text { tex } \times 1 \times 2 \\
110 \text { tex } \times 1 \times 3\end{array}$ & $\begin{array}{l}0.42 \\
0.40\end{array}$ & 0.41 & $\begin{array}{l}20 \\
20\end{array}$ & $\begin{array}{l}0.010 \\
0.011\end{array}$ & $\begin{array}{l}2.38 \\
2.72\end{array}$ \\
\hline
\end{tabular}




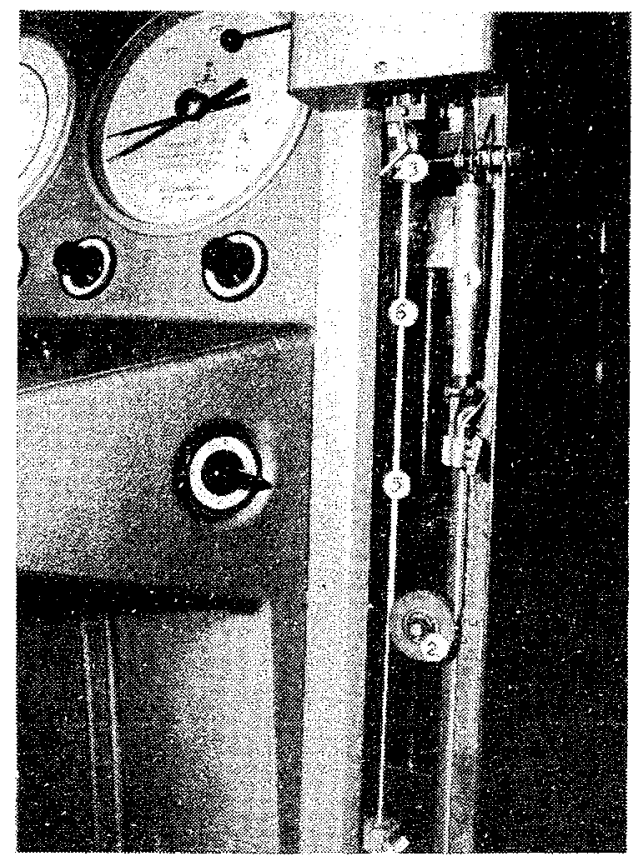

Fig. 1. A measuring unit constructed for studying the yarn-on-yarn friction coefficient' 1) spring dynamometer, 2) block, 3) upper clamp of tensile testing machine, 4) tensile testing machine dynamometer, 5) yarn $A, 6$ ) yarn $B$

vely). A significant difference is noted also between stylon and enkalon in this respect. Within the 23 tex $\times 1 \times 2$ yarn group, the same mean value of friction coefficients is noted for stylon and enkalon (0.36), while the coefficient of amilan differs significantly from them. Of the polyamide yarns tested, amilan is distinct in its higher friction coefficient, whereas stylon and enkalon yarns can be regarded as similar in the nature of their surfaces. The surface character of wet yarns undergoes changes and the obtained values of friction coefficients are lower (Table 3). On the other hand, wet polyester yarns exhibit higher coefficients, which points to different mechanic properties of this group of yarns as well as to a different nature of changes induced by soaking.

In case of netting materials, the results of "wet" measurements are particularly important because of conditions of the fishing gear exploitation. As seen from the data collected in Table 3 , the polyamide yarn friction coefficient decreases significantly on wet, so does the knot resistance (by ca 50\%) (Sadowska, 1976). This fact creates a problem both to the producer and user of netting materials and calls for further research aimed at improving their quality.

The comparison was made between the data obtained in the knot slip resistance analysis and those supplied by tests on the yarn surface character. For the comparative 
Analysis of variance table for yarn friction coefficients

\begin{tabular}{|c|c|c|c|c|c|c|}
\hline \multicolumn{2}{|c|}{ Sort of yarn } & $\begin{array}{l}\text { Source of } \\
\text { variation }\end{array}$ & $\begin{array}{c}\text { Degrees of } \\
\text { freedom }\end{array}$ & Variance & $\begin{array}{c}\text { F test } \\
\text { (calcul.) }\end{array}$ & $\begin{array}{l}\text { F test } \\
\text { (from } \\
\text { tables) }\end{array}$ \\
\hline & & 2 & 3 & 4 & 5 & 6 \\
\hline $\begin{array}{l}\text { stylon NL } \\
\text { enkaton } \\
\text { amilan } \\
\text { torlen }\end{array}$ & $\begin{array}{l}23 \text { tex } \times 1 \times 2 \\
23 \text { tex } \times 1 \times 2 \\
23 \text { tex } \times 1 \times 2 \\
28 \text { tex } \times 1 \times 2\end{array}$ & $\begin{array}{l}\text { between-group } \\
\text { within-group }\end{array}$ & 76 & $\begin{array}{l}0.0106 \\
0.00057\end{array}$ & 17.67 & $\begin{array}{l}\mathrm{F}_{0,05}=2.72 \\
\mathrm{~F}_{0.01}=4.04\end{array}$ \\
\hline $\begin{array}{l}\text { stylon NL } \\
\text { enkalon } \\
\text { amilan } \\
\text { torlen }\end{array}$ & $\begin{array}{r}23 \text { tex } \times 4 \times 3 \\
23 \text { tex } \times 4 \times 3 \\
23 \text { tex } \times 4 \times 3 \\
110 \text { tex } \times 1 \times 3\end{array}$ & $\begin{array}{l}\text { between-group } \\
\text { within-group }\end{array}$ & $\begin{array}{r}3 \\
76\end{array}$ & $\begin{array}{l}0.0061 \\
0.00008\end{array}$ & 76.25 & $\begin{array}{l}\mathrm{F}_{0.05}=2.72 \\
\mathrm{~F}_{0.01}=4.04\end{array}$ \\
\hline $\begin{array}{l}\text { stylon NL } \\
\text { enkalon } \\
\text { amilan }\end{array}$ & $\begin{array}{l}23 \text { tex } \times 8 \times 3 \\
23 \text { tex } \times 8 \times 3 \\
23 \text { tex } \times 8 \times 3\end{array}$ & $\begin{array}{l}\text { between-group } \\
\text { within-group }\end{array}$ & $\begin{array}{r}2 \\
57\end{array}$ & $\begin{array}{l}0.0022 \\
0.00013\end{array}$ & 16.923 & $\begin{array}{l}F_{0.05}=3.15 \\
F_{0.01}=4.98\end{array}$ \\
\hline
\end{tabular}


Table 5

Duncan's procedure-determined significance of differences between yarn friction coefficients

\begin{tabular}{|c|c|c|c|c|c|c|c|}
\hline \multirow{2}{*}{\multicolumn{2}{|c|}{ Sort of yarn }} & \multirow{3}{*}{$\begin{array}{c}\begin{array}{c}\text { Mean } \\
\text { friction } \\
\text { coefficient }\end{array} \\
2\end{array}$} & & \multirow{2}{*}{\multicolumn{2}{|c|}{ Differences of means }} & \multicolumn{2}{|c|}{ Limiting values } \\
\hline & & & & & & $s=0005$ & \\
\hline & 1 & & 3 & 4 & 5 & 6 & 7 \\
\hline $\begin{array}{l}\text { torlen } \\
\text { amilan } \\
\text { enkalon } \\
\text { stylon NL }\end{array}$ & $\begin{array}{l}28 \text { tex } \times 1 \times 2 \\
23 \text { tex } \times 1 \times 2 \\
23 \text { tex } \times 1 \times 2 \\
23 \text { tex } \times 1 \times 2\end{array}$ & $\begin{array}{l}0.41 \\
0.37 \\
0.36 \\
0.36\end{array}$ & $\begin{aligned} \bar{x}-0.36 & \\
0.05 & (4)^{* *} \\
0.01 & (3)^{*}\end{aligned}$ & $\begin{aligned} \overline{\mathrm{x}}-0.36 & \\
0.05 & (3)^{* *} \\
0.01 & (2)^{*}\end{aligned}$ & $\begin{aligned} \bar{x}-0.37 & \\
0.04 & (2)^{* *}\end{aligned}$ & $\begin{array}{ll}0.01 & (2) \\
0.02 & (3) \\
0.02 & (4)\end{array}$ & $\begin{array}{ll}0.02 & (2) \\
0.02 & (3) \\
0.02 & (4)\end{array}$ \\
\hline $\begin{array}{l}\text { torlen } \\
\text { enkalon } \\
\text { stylon NL } \\
\text { amilan }\end{array}$ & $\begin{array}{r}110 \text { tex } \times 1 \times 3 \\
23 \text { tex } \times 4 \times 3 \\
23 \text { tex } \times 4 \times 3 \\
23 \text { tex } \times 4 \times 3\end{array}$ & $\begin{array}{l}0.39 \\
0.35 \\
0.35 \\
0.35\end{array}$ & $\begin{aligned} & \bar{x}-0.35 \\
& 0.04 \quad(4)^{* *}\end{aligned}$ & $\begin{aligned} \bar{x}-0.35 & \\
0.04 & (3)^{* *}\end{aligned}$ & $\begin{array}{r}\bar{x}-0.35 \\
0.04 \quad(2)^{* *}\end{array}$ & $\begin{array}{ll}0.01 & (2) \\
0.01 & (3) \\
0.01 & (4)\end{array}$ & $\begin{array}{ll}0.01 & (2) \\
0.01 & (3) \\
0.01 & (4)\end{array}$ \\
\hline $\begin{array}{l}\text { amilan } \\
\text { enkalon } \\
\text { stylon NL }\end{array}$ & $\begin{array}{l}23 \text { tex } \times 8 \times 3 \\
23 \text { tex } \times 8 \times 3 \\
23 \text { tex } \times 8 \times 3\end{array}$ & $\begin{array}{l}0.36 \\
0.35 \\
0.34\end{array}$ & $\begin{aligned} x-0.34 & \\
0.02 & (3)^{* *} \\
0.01 & (2)^{*}\end{aligned}$ & $\begin{array}{r}\overline{\mathrm{x}}-0.35 \\
0.01 \quad \text { (2)* }\end{array}$ & & $\begin{array}{ll}0.01 & (2) \\
0.01 & (3)\end{array}$ & $\begin{array}{ll}0.01 & (2) \\
0.01 & (3)\end{array}$ \\
\hline
\end{tabular}

* significant at $\alpha=0.05$

** significant at $\alpha=0.01$ 
Evaluation of significance of differences between mean friction coefficients and between mean knot slip resistances, determined with Duncan's new multiple range procedure

\begin{tabular}{|c|c|c|c|c|c|}
\hline & \multirow{2}{*}{ Sorts compared } & \multicolumn{2}{|c|}{ Yarn friction coefficient } & \multicolumn{2}{|c|}{ Knot slip resistance } \\
\hline & & Evaluation of differences & Higher in rank & Evaluation of differences & Higher in rank \\
\hline & 1 & 2 & 3 & 4 & 5 \\
\hline $\begin{array}{l}\text { stylon NL } \\
\text { stylon NL } \\
\text { enkalon } \\
\text { stylon NL } \\
\text { enkalon } \\
\text { amilan }\end{array}$ & $\begin{array}{l}23 \text { tex } \times 1 \times 2 \\
- \text { amilan } \\
- \text { enkalon } \\
- \text { amilan } \\
- \text { torlen } \\
- \text { torlen } \\
- \text { torlen }\end{array}$ & $\begin{array}{l}\mathrm{i} \\
\mathrm{br} \\
\mathrm{i} \\
\text { wi } \\
\text { wi } \\
\text { wi }\end{array}$ & $\begin{array}{c}\text { amilanu } \\
- \\
\text { amilanu } \\
\text { torlenu } \\
\text { torlenu } \\
\text { torlenu }\end{array}$ & $\begin{array}{l}\text { br } \\
\text { i } \\
\text { wi } \\
\text { wi } \\
\text { wi } \\
\text { wi }\end{array}$ & \begin{tabular}{l}
\multicolumn{1}{c}{-} \\
stylonu \\
amilanu \\
torlenu \\
torlenu \\
torlenu
\end{tabular} \\
\hline $\begin{array}{l}\text { stylon NL } \\
\text { stylon NL } \\
\text { enkalon } \\
\text { stylon NL } \\
\text { enkalon } \\
\text { amilan }\end{array}$ & $\begin{array}{l}23 \text { tex } \times 4 \times 3 \\
- \text { amilan } \\
\text { - enkalon } \\
- \text { amilan } \\
- \text { torlen } \\
- \text { torlen } \\
- \text { torlen }\end{array}$ & $\begin{array}{l}\mathrm{br} \\
\mathrm{br} \\
\mathrm{br} \\
\text { wi } \\
\text { wi } \\
\text { wi }\end{array}$ & $\begin{array}{c}- \\
- \\
- \\
\text { torlenu } \\
\text { torlenu } \\
\text { torlenu }\end{array}$ & $\begin{array}{l}\mathrm{br} \\
\mathrm{br} \\
\mathrm{br} \\
\text { wi } \\
\text { wi } \\
\text { wi }\end{array}$ & $\begin{array}{c}- \\
- \\
- \\
\text { torlenu } \\
\text { torlenu } \\
\text { torlenu }\end{array}$ \\
\hline $\begin{array}{l}\text { stylon NL } \\
\text { stylon NL } \\
\text { enkalon }\end{array}$ & $\begin{array}{l}23 \text { tex } \times 8 \times 3 \\
- \text { amilan } \\
- \text { enkalon } \\
- \text { amilan }\end{array}$ & $\begin{array}{l}\text { wi } \\
\mathrm{i} \\
\mathrm{i}\end{array}$ & $\begin{array}{l}\text { amilanu } \\
\text { enkalonu } \\
\text { amilanu }\end{array}$ & $\begin{array}{l}\mathrm{i} \\
\mathrm{br} \\
\text { wi }\end{array}$ & $\begin{array}{c}\text { amilanu } \\
- \\
\text { amilanu }\end{array}$ \\
\hline
\end{tabular}


purposes the data on significance of differences between friction coefficients of the yarns studied and between knot slip resistances, obtained by means of Duncan's test, are summarised in Table 6 . They clearly indicate the yarn surface nature to affect, in a highly significant way, the knot slip resistance. Of the synthetic yarns used in the netting materials production, the quality of polyester knots is ranked above that of polyester ones, and of the latter group the amilan yarns are the highest in rank. The friction coefficient of these yarns is siginficantly different from the coefficient found for stylon and enkalon yarns.

The studies on determining the knot slip resistance-knot tightening force relationship in various synthetic yarns (Sadowska, 1977) showed the relevant regression curves to indicate a highly significant difference in knot slip reșistance between polyester and polyamide yarns. The fruther analysis shows amilan knots to display a higher resistance to slipage, given the appropriately high tightening force. As shown in the present work, the amilan yarns friction coefficient is highly significantly or significantly different from the coefficients found for stylon and enkalon yarns.

In the present paper the knot slip resistance has been found to be related to the yarn properties, which is further supported by the studies on yarn surface characters, the latter being tested for their significance of differences between mean values using $F$ test and Duncan's procedure (Tables 4 and 5). The firction coefficient significantly influences the knot slip resistance regardless of the magnitude of other indices. A high coefficient of polyester yarns provides a good knot resistance to slippage, the tightening force being considerably lower than it is the case in polyamide yarns (Sadowska, 1977). Thus polyamide yarns, those made of stylon in particular, noteworthy for their high resistance properties, need an inreased friction coefficient to ensure their effective use in net making.

\section{CONCLUSIONS}

1. The knot slip resistance of fishing nets made of synthetic yars depends on yarn properties and on the netting production technology.

2. One of the yarn properties that markedly influences the knot slip resistance is the yarn surface nature: the resistance increases with friction coefficient.

3. The polyester yarn knot slip resistance and friction coefficient are highly significantly different from those indices observed for polyamide yarns. Amilan yarns (in most of the sorts tested) are significantly different from stylon and enkalon ones with respect to their friction coefficient and knot slip resistance. To meet the needs of net making, stylon yarns should have their friction coefficient increased.

\section{REFERENCES}

Dembiński W., 1956: Stabilność tkanin sieciowych z przędzy poliamidowej. [The stablenes of poliamide yarn fishing nets]. Roczniki Nauk Rolniczych, 71-B-1. 
Godek J., 1973: Współczynnik tarcia przędzy. [Friction coefficient in yarn]. Metrologia włókiennicza, t. IV. Wydawnictwo Naukowo-Techniczne, Warszawa.

Grudniewski M., 1973: Pomiary wielkości charakteryzujących powierzchnię wyrobów włókienniczych. [Measuring the quantities describing surfaces of textile products]. Metrologia włókiennicza, t. IV. Wydawnictwo Naukowo-Techniczne, Warszawa.

Kalinowski J., 1962: Zagadnienie trwałości węzłów w sieciach rybackich. [The problem of knots in fishing nets]. Zeszyty Naukowe Politechniki Łódzkiej, nr 45.

Lomakina L.N., 1959: Pročnos't uzlovych soedinenij setesnastnych materialov. Izdatelstvo Rybnoe Chozjaistvo, Moskva.

Polska norma PN-69/P-85036. Wyznaczanie odporności węzłów na przesuwanie. [Determination of knot resistance to moving].

Sadowska Z., 1976: Wpływ rodzaju przędz oraz sposobów wykończeń na odporność na przesuwanie węzłów tkanin sieciowych. [An efect of theyarn kind and finish methods on knot shift resistence in fishing nets]. - Zeszyty Naukowe Akademii Rolniczej w Szczecinie, nr 54.

Sadowska Z., 1977: Analiza odporności na przesuwanie węzłów sieci rybackich z przędz syntetycznych $w$ funkcji siły wiązania oraz numeru przędz. [Fishing net knot slip resistance versus knot tightening force and synthetic yarn number]. - Acta Ichthyologica et Piscatoria, VII, 1.

Translated: mgr Teresa Radziejewska

\section{ZALEŻNOŚĆ ODPORNOŚCI NA PRZESUWANIE WĘZŁÓW TKANIN SIECIOWYCH OD CHARAKTERU POWIERZCHNI PRZĘDZ}

\section{Streszczenie}

W pracy przedstawiono badania nad określeniem wpływu charakteru łowierzchni przędz na odporność węzłów na przesuwanie (wskaźnik trwałości węzłów sieci rybackich). Charakter powierzchni przędz określano współczynnikiem tarcia przędz, którego wartości wyznaczano na specjalnie zaprojektowanym stanowisku badawczym. Analizę otrzymanych wyników badań przeprowadzono w oparciu o testy statystyczne.

Stwierdzono, że charakter powierzchni przędz wyraźnie wpływa na odporność węzłów na przesuwanie. Współczynnik tarcia przędz poliestrowych różni się wysoko istotnie od współczynnika tarcia przędz poliamidowych, co znajđuje odbicie w odporności węzłów na przesuwanie. Przeprowadzone badania wskazują, że dla potrzeb sieciarstwa przędze poliamidowe (w tym. szczególnie stylonowe) wymagają zwiększenia współczynnika tarcia przędz. Wyniki badań mogą być praktycznie wykorzystane w zakresie produkcji przędz sieciowych.

3. Садовска

\section{ЗАВИСИМОСТЬ УСТОЙЧИВОСТИ К СМЕЩЕНИЮ УЗЛОВ СЕТНОГО ПОЛОТНА ОТ ХАРАКТЕРА ПОВЕРХНОСТИ ПРЯЖИ}

$$
P \text { e } 3 \text { 10 M }
$$

В работе представлены результаты исследования на тему определения влияния характера поверхности пряжи на устойчивость узлов к смещению (пока- 
затель прочности узлов рыболовных сетеп̆).

Характер поверхности пряжи определяли при помощи коэффициента трения пряжи, величину которого вычисляли на специально сконструированном устройстве.Анализ полученных результатов исследований провели на основе статистических тестов.

В результате исследований установлено, что характер поверхности прящи в значительной степени влияет на устойчивость узлов к смещепию. Коэф̆фициент трения полиэфирной пряжи существенным образом отличается от коэфф̆ициента трения полиамидной пряжи, что находит своё отражение в устойчивости узлов к смещению.

Проведенные исследования указывают на то, что для нуњд сетевязальной промышленности полиамидные пряжи (а особенно стилоновые) требуют увеличения коэффициента трения пряжи. Результаты исследования могут быть использованы практически в области производства сетной пряжи.

Received: 20 IV 1976 r.

Address:

Dr Zofia Sadowska

Instytut Akwakultury i Techniki Rybackiej AR

71-550 Szczecin, ul. Kazimierza Królewicza 3

Polska-Poland 